\title{
Promoting continuity of care for homeless adults with unmet health needs: The role of brief interventions
}

\author{
Denise Lamanna, Vicky Stergiopoulos, Janet Durbin, Patricia \\ O'Campo, Daniel Poremski, and Joshua Tepper
}

Version Post-print/accepted manuscript

\begin{abstract}
Citation Lamanna D, Stergiopoulos V, Durbin J, O’Campo P, Poremski D, (published version) Tepper J. Promoting continuity of care for homeless adults with unmet health needs: The role of brief interventions. Health Soc Care Community. 2018;26:56-64. https://doi.org/10.1111/hsc.12461.
\end{abstract}

Publisher's Statement This is the peer reviewed version of the following article: Lamanna D, Stergiopoulos V, Durbin J, O'Campo P, Poremski D, Tepper J. Promoting continuity of care for homeless adults with unmet health needs: The role of brief interventions. Health Soc Care Community. 2018;26:56-64 which has been published in final form at $10.1111 / \mathrm{hsc} .12461$. This article may be used for non-commercial purposes in accordance with Wiley Terms and Conditions for Self-Archiving.

How to cite TSpace items

Always cite the published version, so the author(s) will receive recognition through services that track citation counts, e.g. Scopus. If you need to cite the page number of the author manuscript from TSpace because you cannot access the published version, then cite the TSpace version in addition to the published version using the permanent URI (handle) found on the record page.

This article was made openly accessible by $\mathbf{U}$ of ' $T$ Faculty. Please tell us how this access benefits you. Your story matters. 
1 Promoting continuity of care for homeless adults with unmet health needs: The role of brief 2 interventions

\section{Abstract}

5 Promoting timely and continuous care for people experiencing homelessness has been a

6 challenge in many jurisdictions, plagued by access barriers and service fragmentation. As part of

7 a larger programme evaluation, this study used qualitative methods to examine the role of a brief

8 interdisciplinary intervention in supporting continuity of care for this population in a large

9 Canadian urban centre. The intervention provides time-limited case management, primary and

10 psychiatric care, and peer accompaniment to homeless adults with unmet health needs

11 discharged from hospital. Data were collected from 52 study participants between July 2013 and

12 December 2014. Three focus groups were conducted with service providers and people with

13 lived experience of homelessness, and 29 individual, semi-structured interviews were conducted

14 with service users and other key informants. Transcripts were analysed using thematic analysis.

15 Analysis was informed by existing frameworks for continuity of care, while remaining open to

16 additional or unexpected findings. Findings suggest that brief interdisciplinary interventions can

17 promote continuity of care by offering low-barrier access, timely and responsive service

18 provision, including timely connection to long-term services and supports, appropriate

19 individualised services and effective co-ordination of services. Although brief interdisciplinary

20 interventions were perceived to promote access, timeliness and co-ordination of care for this

21 population with complex health and social needs, gaps in the local service delivery context can

22 present persisting barriers to care comprehensiveness and continuity.

23 Key words: brief interventions, continuity of care, homelessness, qualitative research, service

24 co-ordination 


\section{5}

26

27

28

29

30

31

32

33

34

35

36

37

38

39

40

41

42

43

44

45

46

47

48

49

50

51

52

53

54

55

56

\section{What is known about this topic:}

- Brief case management for people experiencing homelessness can increase perceived access to community based care, decrease use of acute care and reduce symptom severity.

- Service providers perceive that working alliance can be developed in the context of brief case management interventions for people experiencing homelessness.

\section{What this paper adds:}

- Diverse stakeholder groups perceive that brief interdisciplinary interventions can support continuity of care for people experiencing homelessness and unmet health needs.

- Continuity of care can be promoted through low-barrier service design relevant to the realities of homelessness, a focus on service user engagement, responsive service provision and timely navigation of the service system.

- Service users and providers perceive that interdisciplinary interventions can promote coordination, timeliness and comprehensiveness of services.

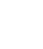

2

3

4

5

6

7

8

9

0

51

2

4

5

6




\section{Introduction}

58 Continuity of care is a complex construct referring to individual patients' experiences of health

59 services as integrated and co-ordinated across settings and over time (Haggerty et al., 2003).

60 Although some variability in definitions persist, it is generally established that continuity of care

61 involves service provision that is longitudinal, timely, accessible, individualised and

62 comprehensive, involving co-ordination and communication between service providers,

63 organisational linkages and lasting interpersonal relationships (Bachrach, 1993; Fortney et al.,

64 2003; Haggerty et al., 2003; Joyce et al., 2004; Vandyk, Graham, Vandenkerkhof, Ross-White,

65 \& Harrison, 2013). In mental health services, it has been associated with improved social

66 functioning, decreased symptom severity and extended periods without readmission to hospital

67 (Puntis, Rugkåsa, Forrest, Mitchell, \& Burns, 2015). However, as continuity of care occurs when

68 separate and discrete elements of care are connected, it is challenging to achieve within the

69 fragmented health and social service systems found in many jurisdictions.

70 Continuity of care is markedly more challenging for individuals with complex support needs

71 (Dixon, Holoshitz, \& Nossel, 2016). Among them, people experiencing homelessness and

72 chronic health conditions warrant particular attention (Fazel, Geddes, \& Kushel, 2014; Gozdzik,

73 Salehi, O’Campo, Stergiopoulos, \& Hwang, 2015; Wagner et al., 2014). In most settings, health

74 service experiences of this population are fragmented and often interrupted, involving extensive

75 barriers to appropriate community-based services (Corrigan, Pickett, Kraus, Burks, \& Schmidt,

76 2015; Gelberg, Browner, Lejano, \& Arangua, 2004; Khandor \& Mason, 2007; Skosireva et al.,

77 2014). Within Canada's publicly funded, single-payer system of universal healthcare coverage,

78 access barriers for people who are homeless include the cost of prescription medications

79 (Hwang, Wilkins et al., 2011; Khandor \& Mason, 2007), service fragmentation, lack of 
80 identifying documents (Campbell, O’Neill, Gibson, \& Thurston, 2015; Krausz et al., 2013), as

81 well as stigma and discrimination (Khandor \& Mason, 2007; Skosireva et al., 2014). Burra,

82 Hwang, Rourke, and Stergiopoulos (2012) found that homeless adults discharged from

83 psychiatric inpatient care in a large Canadian urban centre were less likely to be referred to

84 services than their housed counterparts. Furthermore, the At Home/Chez Soi study found that

85 homeless adults' health service use in five Canadian cities varied by ethno-racial identity,

86 suggesting pervasive disparities (Stergiopoulos et al., 2016). Not surprisingly, homeless adults in

87 Canada utilise costly acute services at disproportionate rates (Hwang, Weaver, Aubry, \& Hoch,

88 2011; Saab, Nisenbaum, Dhalla, \& Hwang, 2016), a trend reported internationally (Buck,

89 Brown, Mortensen, Riggs, \& Franzini, 2012; Department of Health 2010, O'Carroll \& O’Reilly, 90 2008).

91 Despite the success of Housing First interventions in improving housing stability for homeless

92 adults, the model has not been widely implemented across jurisdictions (Aubry et al., 2016;

93 Stergiopoulos, Hwang et al., 2015). Furthermore, although effective health interventions are

94 available in major urban centres, long wait lists for services and strict eligibility criteria create

95 persisting barriers to care continuity, prompting some advocates to call for targeted, low-barrier

96 bridging services to engage and support this disadvantaged population (Hwang \& Burns, 2014;

97 Stergiopoulos, Hwang et al., 2015). Indeed, brief interventions, such as the Critical Time

98 Intervention model (CTI), have been designed to facilitate continuity of care for adults

99 experiencing homelessness, offering timely care while facilitating long-term connections to

100 existing long-term services. Early findings have been promising, suggesting that short-term case

101 management in CTI may improve participants' perceived access to community-based services

102 (Tomita \& Herman, 2015) and reduce rates of psychiatric re-hospitalisation (Shaffer, Hutchison, 
Ayers, Goldberg, \& Herman, 2015; Tomita \& Herman, 2012). A qualitative study of CTI found that case managers perceived that the time-limited model did not hinder development of a positive working alliance (Chen \& Ogden, 2012), widely considered to be crucial in supporting homeless service users (Dixon et al., 2016; Padgett, Henwood, Abrams, \& Davis, 2008). Similarly, O’Toole, Johnson, Borgia, and Rose (2015) found that recipients of a brief health assessment and clinic orientation were 2.5 times more likely to access the clinic within the month. Despite evidence that brief interventions can improve service accessibility and utilisation, their role in promoting other aspects of continuity of care, including comprehensiveness, coordination and appropriateness, has not been sufficiently investigated. Furthermore, service user perspectives have been largely overlooked in previous research.

For both human and economic reasons, improving continuity of care and health outcomes among people who are homeless is a priority in many jurisdictions. Drawing from existing conceptualisations of continuity of care (Bachrach, 1993; Fortney et al., 2003; Haggerty et al., 2003; Joyce et al., 2004; Vandyk et al., 2013), this study aims to explore diverse stakeholder perspectives on the role of brief interventions in supporting continuity of care for this vulnerable population. We engaged service users of a brief interdisciplinary intervention, service providers, service managers and people with lived experience of homelessness to develop a deeper understanding of how brief interventions can support care transitions into long-term services and service users' ongoing connectedness to care.

\section{Description of the Intervention}

Informed by earlier research into brief interventions for adults experiencing homelessness, Coordinated Access to Care for Homeless People (CATCH) provides brief (up to 6 months) case management and access to interdisciplinary care for homeless adults discharged from hospital 
126 and lacking appropriate health and social supports (Stergiopoulos et al., 2017). "Homeless" is

127 here defined as living on the street, in a crisis/emergency shelter or couch surfing. The brief

128 intervention operates in Toronto, Canada, and is a partnership of three local hospitals, a large

129 community mental health agency, a homeless shelter, a physician practice plan and a peer-run

130 service organisation.

131 The intervention aims to provide time-limited services while establishing continuity of care

132 through timely referral to and co-ordination of appropriate and comprehensive long-term health

133 and social services. Three CATCH case managers (CMs), each with a caseload of approximately

13420 service users, serve homeless adults discharged from one of three urban hospitals. CMs assess

135 needs, introduce service users to providers within the interdisciplinary team, offer assertive

136 outreach and home visits, and facilitate connections to income supports, housing, and

137 community-based health and social services as needed. Furthermore, CATCH offers service

138 users a dedicated weekly shelter-based clinic staffed by a nurse, a primary care physician and

139 two psychiatrists, helping address immediate health needs while seeking long-term services.

140 Peers accompany service users to clinic and other appointments in the community as needed.

141 Monthly team meetings serve to review comprehensive care and crisis plans. Additional

142 programme partnerships facilitate timely access to income supports, long-term case management

143 and comprehensive primary healthcare.

\section{Methods}

145 This work is a sub-study within a larger mixed methods evaluation of the CATCH programme 146 investigating health and service use outcomes among 225 programme participants over 6 months

147 of follow-up (Stergiopoulos et al., 2017). We chose mixed methods and a realist stance,

148 particularly useful for its strength in linking quantitative measures of programme effectiveness 
149 with qualitative inquiry to expose complex social and structural factors (Pawson, Greenhalgh,

150 Harvey, \& Walshe, 2005). Critical realism can be particularly useful in examining the role of

151 health interventions for socially marginalised populations, where the outcome of an intervention

152 must be specified through both mechanisms and contexts (Frauley \& Pearce, 2007). In this sub-

153 study, drawing from existing conceptualisations of continuity of care (Bachrach, 1993; Haggerty

154 et al., 2003; Vandyk et al., 2013), we examine diverse stakeholder perspectives on the role of a

155 brief intervention in supporting continuity of care, using qualitative methods for their strength in

156 studying phenomena that have not been well documented (Miles, Huberman, \& Saldaña, 2014;

157 Palinkas et al., 2015). We conducted qualitative interviews and focus groups, and analysed data

158 using thematic analysis (Braun \& Clarke, 2006). Our study received research ethics board

159 approval from St. Michael's Hospital in Toronto, Canada.

\section{Participant sample and recruitment}

161 We sampled 52 participants, including programme service users $(\mathrm{N}=22)$, programme staff $(\mathrm{N}=$ 162 8), managers of partnered organisations $(\mathrm{N}=7)$, people with prior lived experience (PWLE) of 163 homelessness and mental health conditions $(\mathrm{N}=8)$, and external service providers who work in 164 the area of homelessness and mental health or substance misuse and are not connected to the 165 programme under study $(\mathrm{N}=7)$. We purposefully selected stakeholders especially

166 knowledgeable about the subject area, forming a sample that encompassed a range of 167 perspectives on both the brief intervention itself and the broader context of homeless service 168 provision. The triangulation of data sources, including programme service users, programme 169 staff and managers, and individuals external to the programme, expanded the range of 170 perspectives in which we looked for patterns and supported the trustworthiness of our findings 171 (Miles et al., 2014). 
172 We recruited service user participants at the 6-month follow-up interview of a study evaluating 173 programme outcomes. Thirty-three service users among 225 service users participating in the

174 programme's outcome evaluation were randomly selected and invited to complete a qualitative

175 interview at the 6-month follow-up visit. Twenty-two of the 33 service user participants

176 approached (66\%) agreed to participate. Table 1 presents the characteristics of service users

177 completing qualitative interviews. All partner organisations' managers were invited to participate 178 in individual interviews, and all organisations were represented. All CATCH programme staff 179 were invited to participate, and only one staff member was unable to attend. PWLE were 180 recruited through an affiliated hospital's consumer advisory panel, and external providers were 181 recruited through organisations known to work in this sector. Written informed consent was 182 obtained from all study participants by trained research staff. Service users and PWLE received $183 \$ 25$ honoraria and public transportation fares for their participation.

\section{Data collection and analysis}

185 Data collection took place between July 2013 and December 2014 in Toronto, Canada.

186 Programme staff, external service providers and PWLE participated in three semi-structured 187 focus groups. Programme service users and managers of partnered organisations participated in 18829 semi-structured individual interviews. All interview and focus group guides solicited "thick" 189 descriptions of the topic, including information on health and social service needs of this 190 population, service availability and accessibility, processes that enable timely access to 191 appropriate, comprehensive services and co-ordination of services across different service 192 sectors. Guides for service user participants also explored experiences with the brief intervention, 193 including direct service provision and transfers to long-term providers. Guides for programme 194 staff and managers solicited additional information on the intervention, contextualized within the 
195 larger service system; those for external providers and PWLE focused on their perspectives of 196 the service system's capacity to serve people experiencing homelessness. The service user 197 interview guide was piloted with three programme service users prior to data collection. The 198 interviewers and lead researcher met regularly throughout data collection to refine the interview 199 guides and prompts in an iterative process to better understand participant perspectives (Miles et 200 al., 2014). Two interviewers experienced with disadvantaged populations were responsible for all 201 data collection; both facilitated each focus group, and one or both conducted each individual 202 interview. Both interviewers had previously met and established rapport with service user 203 participants. Consistency of interviews was promoted by interviewer training and review of early 204 transcripts. The focus groups and interviews were audio-recorded and transcribed verbatim. The 205 focus groups had a mean length of $86 \mathrm{~min}$, and interviews had a mean length of $59 \mathrm{~min}$. The 206 sample size provided access to rich data and in-depth descriptions of care experiences.

207 Data were analysed using thematic analysis, informed by conceptual work outlined earlier, 208 including the accessibility, timeliness, lasting therapeutic relationships, co-ordination and 209 comprehensiveness of services, while remaining open to additional or unexpected findings 210 (Bachrach, 1993; Braun \& Clarke, 2006; Haggerty et al., 2003; Vandyk et al., 2013).

211 During data analysis, we looked for and highlighted consistencies and divergences between 212 diverse participant groups' perspectives. To further enhance methodological rigour, we pursued 213 analyst triangulation (Miles et al., 2014). The first interviewer coded a subset of transcripts, 214 which were reviewed by the second interviewer, and discussion informed minor revisions to the 215 emerging coding framework. The first interviewer coded the remaining transcripts. Upon 216 completion of coding, the full code list and a sample of relevant quotes were reviewed by the 217 first interviewer, lead researcher and an additional research team member in an iterative process 
218 that included review of emerging themes and generation of a thematic map of the analysis

219 (Braun \& Clarke, 2006), refinement of themes, and grouping into themes and sub-themes. Data

220 analysis was facilitated by qualitative data management software QSR NVivo vers.9.2.

\section{Findings}

222 Study participants across stakeholder groups identified several key elements promoting

223 continuity of care within the brief intervention, including elements promoting low-barrier access

224 to services, timeliness, individualised and engaging care, service navigation and co-ordination.

225 Systemic issues of service availability were also exposed. These themes are elaborated below 226 with illustrative quotes.

\section{$227 \quad$ Facilitating access to services}

228 Participants identified that service location and appointment escorts were key components

229 promoting continuity of care. Service users valued that CMs were "willing to go out in the

230 community" (service user 6), and that the weekly clinic was easily accessed by public transit. As

231 one service user commented, "one streetcar, [and] I'm there” (service user 12). Another service

232 user valued that the weekly clinic was located "close to my other appointments ... I'm down here

233 a lot" (service user 19).

234 Programme staff and service users perceived that accompaniments to appointments with new

235 service providers were effective in reducing barriers to accessing services. Cognitive

236 impairments or active mental health symptoms made it difficult for some participants to

237 remember or make it to appointments, while others were nervous about meeting new service

238 providers. PWLE similarly reported that their mental health sometimes made it challenging to

239 meet their scheduled meetings, with one saying that a one-hour appointment consumes "half the 
240 day ... it's just dealing with my disorder to get there”. Accompaniments by peer support workers

241 or CMs offered practical and emotional support, and improved appointment attendance: "I

242 needed somebody just to start me off going to appointments, and I was feeling weak and worried

243 and anxious, so [CM] hooked me up a couple of times with that" (service user 22).

\section{Offering timely services}

245 Within the brief intervention, service users were offered immediate access to the

246 interdisciplinary team's range of skills and expertise. Such timely service connections were a

247 new experience for most service users, as this quote highlights: "I was not expecting it to be so

248 quick ... that was amazing" (service user 3). Service users described timeliness, through both

249 scheduled appointments and drop-in clinic times, as highly valued.

250 Both service users and providers perceived that some health and social needs could not be met by

251 the brief intervention's own clinicians. Promptly scheduled appointments with additional

252 services were therefore deemed necessary to effectively address service user needs. Timely

253 connections to needed supports alleviated service users' stress, as this service user, quickly

254 approved for income support, reported: "I didn’t expect it that quickly ... I was relieved" (service

255 user 9). Another service user, referred to a specialist, described: "I can't believe how quickly I

256 got in to see the rheumatologist ... it's best to get it done really quickly, so I don't have to worry

257 about it" (service user 21).

258 The importance of timeliness extended beyond the value of simply receiving treatment promptly.

259 Quick connections to appropriate services signified rapid results, and some service users needed

260 to see results to sustain the hopefulness and motivation to remain engaged with the help-seeking

261 process. As shown above, many service users described the speed of service connections to 
262 programme and community-based service providers with enthusiasm and surprise. Some service

263 users explicitly contrasted these experiences with past ones in which long wait times eroded

264 hopefulness, as this individual did in describing his past searches for services:

271 Supporting early and sustained engagement

272 Efforts to support early and sustained engagement by attending to individual service user needs, 273 maintaining frequent contact, and offering knowledgeable and welcoming services emerged as

274 critical to supporting continuity of care. Identifying service users' individual needs, essential to 275 planning appropriate and lasting long-term service connections, was not always a straightforward

276 process. Although some service users easily shared information with their CMs, others

277 considered the relationship-building process challenging. One service user feared stigmatisation

278 and "didn't want to dump everything at once" (service user 2). Another service user reported

279 feeling uncomfortable with her CM "at first, because she was a stranger" and took "four or five

280 visits ... just getting to know her" before a comfortable rapport developed (service user 1).

281 Nearly all service users described that once confidence and trust developed, communicating their

282 needs became easier. In fact, a number of service users felt responsible for "honesty [about] what

283 your issues are" in order to promote their own successful service connections (service user 14).

284 Service users described that when they were seen as individuals with personal needs and goals, 285 they were more likely to engage with the brief intervention and were more satisfied with the 286 referrals made to long-term services, as highlighted below: 
$[\mathrm{CM}]$ really listened to my needs. At detox, they just always have the same solution, which is, put the person in a recovery home for a year or two ... that's what they do, right? It's not about, "what does this person as an individual need?" ... She was open to what my needs were in the moment (service user 6).

Additionally, CMs kept service users engaged through regular progress updates on the status of referrals to long-term services and by responding to service user questions promptly. As this service user described: “[CM] checked in with me through text: 'Everything's okay, this is an update' [or] 'nothing has changed', you know. That was cool, like I didn't go days on end wondering... 'is he just screwing me around?'” (service user 11).

Programme staff perceived that their deep understanding of individual and systemic factors associated with homelessness facilitated service users' early and sustained engagement and care continuity:

Everyone here has a good knowledge of homeless issues. Like, the short-term and long-term issues that affect our service users. So, it's very specialised and that's an important piece ... we create a comfortable environment, so service users feel comfortable, at ease. They develop quick, easy rapport with us (programme staff).

Relatedly, nearly all PWLE, service users and frontline service providers perceived that service providers' welcoming and respectful approach can support service engagement and continuity of care. Exposing the prevalence of interpersonal barriers in the mental healthcare system, one PWLE shared, "I have a difficulty with psychiatrists. I really find they're not very down-toearth". This struck a chord with other PWLE focus group participants, who added, "not really easy to talk to them". Within the brief intervention, many service users found that staff's sincere and welcoming interpersonal approach strengthened engagement and the working alliance, as this service user highlighted: "[the CM had a] wonderful personality ... that makes you want to establish a bond with her. So certainly, it's easy to have a working relationship with her" (service user 7). 


\section{Co-ordinating a multitude of services}

314 Co-ordination of diverse services can facilitate access, reduce unnecessary duplication, and 315 improve the effectiveness and efficiency of health and social services (Bodenheimer, 2008).

316 Service co-ordination in the CATCH programme involved managing new and existing service 317 connections to ensure congruence in service goals, and clear, complementary roles for service 318 providers. Within the interdisciplinary brief intervention, service co-ordination was facilitated by

319 frequent communication between providers and shared emphasis on flexible service provision.

320 Intervention staff and physicians felt they "work very individually, but well as a team". At the

321 same time, some service users entered the brief intervention with one or more pre-existing

322 service connections, requiring additional co-ordination efforts to support continuity of care. This

323 process entailed identifying all pre-existing service providers, streamlining redundant services,

324 and organizing care from multiple specialties and sectors. For example, one staff person reported

325 that "part of our job [is] to get an idea of how many different doctors they're seeing, and-yeah,

326 it's a little bit like investigation work sometimes".

327 Service users highly valued the navigation of the service system and co-ordination of services

328 provided by their CMs. Reflecting on the time of their enrolment to the programme, many

329 service user participants described feeling overwhelmed by the large number of their own unmet

330 needs and ill-equipped to independently manage co-ordinating needed services. Service users felt

331 that CMs' advocacy and support were instrumental in their progress. This was highlighted by

332 one service user who was able to access medication following information transfer by his CM:

It was nice to have that backup support, where she could call and [tell the agency], user 14). 
Staff of the brief intervention similarly perceived that their position and knowledge of the service system made them, at times, more effective than service users themselves in co-ordinating longterm services:

Programme staff 1: We have more of a voice than if a client calls themselves... Programme staff 2: And because we've done it a hundred times and they haven't. Programme staff 1: We know the system.

\section{The service landscape}

For brief interventions to promote continuity of care, appropriate services must be available within the service system. Services for some conditions were readily available; however, all participants employed in the service system, as well as a smaller number of service user and PWLE participants, identified that other supports were scarce. These constraints in the service system limited the comprehensiveness of immediate as well as long-term services attainable. Constraints extended to health services that were essential for this population, including traumatic brain injury services and mental healthcare. Echoing many participants in this study, an external service provider perceived it was becoming "harder and harder to secure psychiatrists to work with people". Similarly, the limited availability of affordable housing presented a significant challenge, as one programme manager highlighted:

When the housing stock is not there, when rent is going through the roof every year, it is just almost impossible ... the societal difficulties are really very tough. So, we try to do our best, but the problems are still there (manager).

Furthermore, system fragmentation and service silos led programme staff to preferentially refer service users to long-term service agencies offering integrated health and social services for vulnerable populations. Staff perceived that once service users were connected to one service at a multi-service agency, linkages within the agency would facilitate in-house referrals for service users' additional or evolving care needs. The effectiveness of this approach was supported by some service users, as those connected to integrated-service agencies perceived easy access to 
364 multiple services. When asked about these long-term services, service users said "they do

365 everything for me. If a blessing is happening, let's keep it happening" (service user 22) and

366 pointed to the "one stop shopping. There's so much available there that I might need that—why

367 go to ten, five different doctors when you [can] go to one office?" (service user 9).

\section{Discussion}

369 Our findings highlight and expose key elements promoting continuity of care for homeless 370 people with unmet health needs, including experiences of access, timeliness, co-ordination,

371 comprehensiveness and relationships in the context of a brief interdisciplinary intervention.

372 Study findings suggest that brief interventions can promote continuity of care through low-

373 barrier design relevant to the realities of homelessness. These design features can include timely

374 service provision, intentional efforts to support service user engagement, including the

375 interpersonal characteristics of service providers, and helping service users navigate the service

376 system. We also found that systemic issues of constrained service availability can limit the

377 comprehensiveness of services, although multi-service agencies with integrated case

378 management, primary and mental healthcare were perceived to mitigate these challenges. Our

379 findings support earlier research on long-term service provision and add a multi-stakeholder 380 perspective.

381 An ample body of literature has documented the many barriers to services faced by people 382 experiencing homelessness and called for flexible, accessible designs in brief and long-term 383 programmes (O’Toole et al., 2015; Padgett et al., 2008; Stergiopoulos et al., 2016). Consistent 384 with the literature, we found that continuity of care was enhanced by the accessibility of the brief 385 intervention, particularly related to service location. Additionally, our findings suggest that peer 386 accompaniment to appointments may be a useful strategy in supporting continuity of care. As 
387 appointment absenteeism is a significant issue in service provision for homeless people (Hwang,

388 Wilkins et al., 2011; Weiser et al., 2006), peer accompaniment may have an important role to

389 play. The mental health services literature indicates that peers can promote service users' social

390 functioning, sense of empowerment and hope for a better future (Repper \& Carter, 2011), and

391 may be well-positioned to support this population.

392 Timeliness of services was identified as a key element supporting continuity of care, similar to

393 studies of homeless persons' experiences with long-term services. However, whereas participants

394 in long-term services often identify the importance of timeliness through their negative

395 experiences with wait lists (Corrigan et al., 2015; Gelberg et al., 2004), participants in this brief

396 intervention did so through their positive experiences with immediate service provision. Our

397 findings highlight that timely service provision within the brief intervention and through referrals

398 to long-term services led to prompt attention to acute and chronic health conditions, relieved

399 stress, and encouraged hopefulness and motivation to sustain engagement in the help-seeking

400 process. Conceptual models of recovery consistently highlight the importance of hope and goal-

401 setting (Leamy, Bird, Boutillier, Williams, \& Slade, 2011; Mead \& Copeland, 2000), and

402 evidence indicates that internal motivation is an important predictor of reduced substance use

403 (Collins, Malone, \& Larimer, 2012) and mental health service utilisation (Jakupcak et al., 2013).

404 While there is little evidence supporting any specific strategies to increase hopefulness, some

405 qualitative work has suggested that experiences of success are generally helpful (Schrank, Bird,

406 Rudnick, \& Slade, 2012). It is noteworthy that, among homeless people seeking health services,

407 brief interventions may promote hope and motivation by providing more timely service

408 connections. 
409 Service users highly valued providers' responsiveness, service co-ordination and advocacy. Their 410 complex needs required multiple service connections, and co-ordination could be a challenging 411 process, especially when not all pre-existing service connections were disclosed. The finding of 412 perceived effective co-ordination of services by a brief intervention resonates with literature on 413 the CTI model of time-limited case management, which offers up to 9 months of case

414 management to individuals with mental health problems transitioning from institutional to 415 community settings (Herman, Conover, Felix, Nakagawa, \& Mills, 2007). Although research on 416 its effectiveness is largely restricted to settings in the US, CTI has been associated with easier 417 access to services (Tomita \& Herman, 2015) and more regular outpatient service utilisation 418 (Dixon et al., 2009).

419 Although some service users found it challenging to communicate their needs and preferences, it 420 was a necessary step towards individualized service plans and appropriate service connections.

421 The value of relationships, interpersonal characteristics of friendliness and respectfulness, and 422 knowledge of the issues associated with the "realities of homelessness" emerged as key 423 supporting elements. These qualities facilitated service user engagement and participation in the 424 planning process (Dixon et al., 2016). As a result, care plans aligned with service user goals, a 425 recognised feature in the promotion of continuity of care in many long-term services (Corrigan et 426 al., 2012, Mental Health Commission of Canada 2009). Service provider training to enable care 427 that is accessible, engaging and appropriate for people experiencing homelessness is an 428 important consideration across the service system.

429 In the context of a brief intervention, longitudinality, the long-term connection of service users to 430 care, was facilitated by rapid service connections to long-term services. To that end, we found 431 that both within the brief intervention and long-term community services, the value of 
432 interdisciplinary models including some combination of primary, psychiatric and social services

433 emerged strongly. Programme partnerships within the brief interdisciplinary intervention

434 improved capacity to meet several service user care needs quickly; this "one stop shopping"

435 promoted continuity of care through more comprehensive and co-ordinated service provision.

436 This finding is consistent with work by O'Toole, Johnson, Aiello, Kane, and Pape (2016), which

437 found clinics serving homeless populations may be more effective when appropriate social

438 services are co-located. There is an international push for service hubs and integrated primary

439 and mental health services, often referred to as "integrated" or "collaborative care" models

440 (Kaliebe, 2016; Woltmann et al., 2012). For people with mental health conditions that are

441 currently or recently homeless, integrated services have been associated with improvements in

442 community functioning, utilisation of community health services (Stergiopoulos, Schuler et al.,

4432015 ) and treatment adherence (Ho et al., 2015; Parashar et al., 2011). When combined with

444 housing, integrated services are generally preferred by people experiencing homelessness and

445 mental health challenges (Corrigan et al., 2015; Lee, Crowther, Keating, \& Kulkarni, 2013).

446 However, comprehensive health and social services must be available across the system for

447 continuity of care to be achieved (Bachrach, 1993), and this study draws attention to a systemic

448 lack of affordable housing and specialised health services frequently required by the homeless

449 population, including Housing First programmes. Another Canadian study of homeless and

450 precariously housed older adults similarly found that limited availability of housing and

451 accessible community services created a context of constrained options for service users and

452 those trying to support them (Ploeg, Hayward, Woodward, \& Johnston, 2008). There is an urgent

453 need to address existing service gaps, including appropriate affordable housing, health, mental

454 health and rehabilitative services, in many jurisdictions facing similar challenges. As 
455 homelessness is a social phenomenon partially created and perpetuated by systemic inequities

456 (Hwang \& Burns, 2014), these issues cannot be overcome by interventions targeting problems at 457 the individual level.

\section{$458 \quad$ Limitations}

459 The current study has some limitations. As two-thirds of service users approached agreed to 460 participate in this study, we may have missed the perspectives of some service user participants 461 whose experiences differed from those of our sample. While earlier work has found that 462 demographic characteristics including gender and ethno-racial identities impact homeless 463 persons' experiences accessing healthcare (Gelberg et al., 2004; Stergiopoulos et al., 2016), these 464 diverse experiences did not emerge in our data. Additionally, service users within different 465 service delivery settings may differ in their experiences of continuity of care. However, our 466 purpose was to generate knowledge on the local intervention and inform modifications to this 467 and similar models of care delivery. Despite these limitations, few studies have examined 468 elements of brief interventions supporting continuity of care for people experiencing 469 homelessness. Our findings therefore may be helpful to planners and service providers engaged 470 in the design and delivery of appropriate services for this population.

\section{Conclusions}

472 Within fragmented and poorly resourced service delivery systems, our findings suggest that brief

473 targeted interventions have an important role in promoting continuity of care for homeless

474 people with complex health needs. Future efforts to improve continuity of care and health

475 outcomes for this population should consider brief interdisciplinary models and evaluate a range

476 of brief interventions suitable to diverse service delivery contexts. 
Aubry, T., Goering, P., Veldhuizen, S., Adair, C. E., Bourque, J., Distasio, J., ... Tsemberis, S. (2016). A multiple-city RCT of Housing First with Assertive Community Treatment for homeless Canadians with serious mental illness. Psychiatric Services, 67, 275-281.

Bachrach, L. (1993). Continuity of care and approaches to case management for long-term mentally ill patients. Hospital and Community Psychiatry, 44, 465-468.

Bodenheimer, T. (2008). Coordinating care-A perilous journey through the health care system. New England Journal of Medicine, 358, 1064-1071.

Braun, V., \& Clarke, V. (2006). Using thematic analysis in psychology. Qualitative Research in Psychology, 3, 77-101.

Buck, D. S., Brown, C. A., Mortensen, K., Riggs, J. W., \& Franzini, L. (2012). Comparing homeless and domiciled patients' utilization of the Harris County, Texas public hospital system. Journal of Health Care for the Poor and Underserved, 23, 1660-1670.

Burra, T. A., Hwang, S. W., Rourke, S. B., \& Stergiopoulos, V. (2012). Homeless and housed inpatients with schizophrenia: Disparities in service access upon discharge from hospital. International Journal of Mental Health and Addiction, 10, 778-789.

Campbell, D. J., O’Neill, B. G., Gibson, K., \& Thurston, W. E. (2015). Primary healthcare needs and barriers to care among Calgary's homeless populations. BMC Family Practice, 16, 139.

Chen, F.-P., \& Ogden, L. (2012). A working relationship model that reduces homelessness among people with mental illness. Qualitative Health Research, 22, 373-383.

Collins, S. E., Malone, D. K., \& Larimer, M. E. (2012). Motivation to change and treatment attendance as predictors of alcohol-use outcomes among project-based housing first residents. Addictive Behaviors, 37, 931-939.

Corrigan, P., Angell, B., Davidson, L., Marcus, S. C., Salzer, M., Kottsieper, P., ... Stanhope, V. (2012). From adherence to self-determination: Evolution of a treatment paradigm for people with serious mental illnesses. Psychiatric Services, 63, 169-173.

Corrigan, P., Pickett, S., Kraus, D., Burks, R., \& Schmidt, A. (2015). Community-based participatory research examining the health care needs of African Americans who are homeless with mental illness. Journal of Health Care for the Poor and Underserved, 26, 119-133.

Department of Health (2010). Healthcare for single homeless people. London: National Health Service.

Dixon, L., Goldberg, R., Iannone, V., Lucksted, A., Brown, C., Kreyenbuhl, J., ... Potts, W. (2009). Use of a Critical Time Intervention to promote continuity of care after psychiatric inpatient hospitalization. Psychiatric Services, 60, 451-458.

Dixon, L., Holoshitz, Y., \& Nossel, I. (2016). Treatment engagement of individuals experiencing mental illness: Review and update. World Psychiatry, 15, 13-20.

Fazel, S., Geddes, J. R., \& Kushel, M. (2014). The health of homeless people in high-income countries: Descriptive epidemiology, health consequences, and clinical and policy recommendations. Lancet, 384, 1529-1540.

Fortney, J., Sullivan, G., Williams, K., Jackson, C., Morton, S. C., \& Koegel, P. (2003). Measuring continuity of care for clients of public mental health systems. Health Services Research, 38, 1157-1175. 
Frauley, J., \& Pearce, F. (2007). Critical realism and the social sciences: Methodological and epistemological preliminaries. In J. Frauley, \& F. Pearce (Eds.), Critical realism and the social sciences: Heterodox elaborations (3-29). Toronto: University of Toronto Press.

Gelberg, L., Browner, C. H., Lejano, E., \& Arangua, L. (2004). Access to women's health care: A qualitative study of barriers perceived by homeless women. Women and Health, 40, $87-100$.

Gozdzik, A., Salehi, R., O’Campo, P., Stergiopoulos, V., \& Hwang, S. W. (2015). Cardiovascular risk factors and 30-year cardiovascular risk in homeless adults with mental illness. BMC Public Health, 15, 165.

Haggerty, J., Reid, R., Freeman, G., Starfield, B., Adair, C., \& Mckendry, R. (2003). Continuity of care: A multidisciplinary review. BMJ, 327, 1219-1221.

Herman, D., Conover, S., Felix, A., Nakagawa, A., \& Mills, D. (2007). Critical Time Intervention: An empirically supported model for preventing homelessness in high risk groups. Journal of Primary Prevention, 28, 295-312.

Ho, S. B., Brau, N., Cheung, R., Liu, L., Sanchez, C., Sklar, M., ... Groessl, E. J. (2015). Integrated care increases treatment and improves outcomes of patients with chronic Hepatitis $\mathrm{C}$ virus infection and psychiatric illness or substance abuse. Clinical Gastroenterology and Hepatology, 13, 2005-2014.

Hwang, S. W., \& Burns, T. (2014). Health interventions for people who are homeless. Lancet, $384,1541-1547$.

Hwang, S. W., Weaver, J., Aubry, T., \& Hoch, J. S. (2011). Hospital costs and length of stay among homeless patients admitted to medical, surgical, and psychiatric services. Medical Care, 49, 350-354.

Hwang, S. W., Wilkins, E., Chambers, C., Estrabillo, E., Berends, J., \& Macdonald, A. (2011). Chronic pain among homeless persons: Characteristics, treatment, and barriers to management. BMC Family Practice, 12, 73-81.

Jakupcak, M., Hoerster, K. D., Blais, R. K., Malte, C. A., Hunt, S., \& Seal, K. (2013). Readiness for change predicts VA mental healthcare utilization among Iraq and Afghanistan war veterans. Journal of Traumatic Stress, 26, 165-168.

Joyce, A. S., Wild, T. C., Adair, C. E., McDougall, G. M., Gordon, A., Costigan, N., ... Barnes, F. (2004). Continuity of care in mental health services: Toward clarifying the construct. Canadian Journal of Psychiatry, 49, 539-550.

Kaliebe, K. E. (2016). The future of psychiatric collaboration in federally qualified health centers. Psychiatric Services, 67, 827-829.

Khandor, E., \& Mason, K. (2007). The street health report 2007. Toronto: Street Health.

Krausz, R. M., Clarkson, A. F., Strehlau, V., Torchalla, I., Li, K., \& Schuetz, C. G. (2013). Mental disorder, service use, and barriers to care among500 homeless people in 3 different urban settings. Social Psychiatry and Psychiatric Epidemiology, 48, 1235-1243.

Leamy, M., Bird, V., Boutillier, C. L., Williams, J., \& Slade, M. (2011). Conceptual framework for personal recovery in mental health: Systematic review and narrative synthesis. British Journal of Psychiatry, 199, 445-452.

Lee, S. J., Crowther, E., Keating, C., \& Kulkarni, J. (2013). What is needed to deliver collaborative care to address comorbidity more effectively for adults with a severe mental illness? Australian and New Zealand Journal of Psychiatry, 47, 333-346.

Mead, S., \& Copeland, M. E. (2000). What recovery means to us: Consumers' perspectives. Community Mental Health Journal, 36, 315-328. 
Mental Health Commission of Canada (2009). Toward recovery and well-being: A framework for a mental health strategy for Canada. Ottawa.

Miles, M. B., Huberman, A. M., \& Saldaña, J. (2014). Qualitative data analysis: A methods sourcebook. Thou sand Oaks: Sage Publications.

O'Carroll, A., \& O'Reilly, F. (2008). Health of the homeless in Dublin: Has anything changed in the context of Ireland's economic boom? European Journal of Public Health, 18, 448453.

O’Toole, T. P., Johnson, E. E., Aiello, R., Kane, V., \& Pape, L. (2016). Tailoring care to vulnerable populations by incorporating social determinants of health: The veterans health administration's "homeless patient aligned care team" program. Preventing Chronic Disease, 13, https://doi.org/10.5888/pcd13.150567

O’Toole, T. P., Johnson, E. E., Borgia, M. L., \& Rose, J. (2015). Tailoring outreach efforts to increase primary care use among homeless veterans: Results of a randomized controlled trial. Journal of General Internal Medicine, 30, 886-898.

Padgett, D. K., Henwood, B., Abrams, C., \& Davis, A. (2008). Engagement and retention in services among formerly homeless adults with co-occurring mental illness and substance abuse: Voices from the margins. Psychiatric Rehabilitation Journal, 31, 226-233.

Palinkas, L., Horwitz, S., Green, C., Wisdom, J., Duan, N., \& Hoagwood, K. (2015). Purposeful sampling for qualitative data collection and analysis in mixed method implementation research. Administration and Policy in Mental Health and Mental Health Services Research, 42, 533-544.

Parashar, S., Palmer, A., O’Brien, N., Chan, K., Shen, A., Coulter, S., ... Hogg, R. (2011). Sticking to it: The effect of maximally assisted therapy on antiretroviral treatment adherence among individuals living with HIV who are unstably housed. AIDS and Behavior, 15, 1612-1622.

Pawson, R., Greenhalgh, T., Harvey, G., \& Walshe, K. (2005). Realist review- A new method of systematic review designed for complex policy interventions. Journal of Health Services Research and Policy, 10, 21-34.

Ploeg, J., Hayward, L., Woodward, C., \& Johnston, R. (2008). A case study of a Canadian homelessness intervention programme for elderly people. Health and Social Care in the Community, 16, 593-605.

Puntis, S., Rugkåsa, J., Forrest, A., Mitchell, A., \& Burns, T. (2015). Associations between continuity of care and patient outcomes in mental health care: A systematic review. Psychiatric Services, 66, 354-363.

Repper, J., \& Carter, T. (2011). A review of the literature on peer support in mental health services. Journal of Mental Health, 20, 392-411.

Saab, D., Nisenbaum, R., Dhalla, I., \& Hwang, S. W. (2016). Hospital readmissions in a community-based sample of homeless adults: A matched-cohort study. Journal of General Internal Medicine, 31, 1011-1018.

Schrank, B., Bird, V., Rudnick, A., \& Slade, M. (2012). Determinants, self-management strategies and interventions for hope in people with mental disorders: Systematic search and narrative review. Social Science and Medicine, 74, 554-564.

Shaffer, S. L., Hutchison, S. L., Ayers, A. M., Goldberg, R. W., \& Herman, D. (2015). Brief Critical Time Intervention to reduce psychiatric rehospitalization. Psychiatric Services, 66, 1155-1161. 
Skosireva, A., O’Campo, P., Zerger, S., Chambers, C., Gapka, S., \& Stergiopoulos, V. (2014). Different faces of discrimination: Perceived discrimination among homeless adults with mental illness in healthcare settings. BMC Health Services Research, 14, 1-11.

Stergiopoulos, V., Gozdzik, A., Nisenbaum, R., Lamanna, D., Hwang, S. W., Tepper, J., \& Wasylenki, D. (2017). Integrating hospital and community care for homeless people with unmet mental health needs: Program rationale, study protocol and sample description of a brief multidisciplinary case management intervention. International Journal of Mental Health and Addiction, 15, 362-378.

Stergiopoulos, V., Gozdzik, A., Nisenbaum, R., Vasiliadis, H.-M., Chambers, C., McKenzie, K., \& Misir, V. (2016). Racial-ethnic differences in health service use in a large sample of homeless adults with mental illness from five Canadian cities. Psychiatric Services, 67, 1004-1011.

Stergiopoulos, V., Hwang, S. W., Gozdzik, A., Nisenbaum, R., Latimer, E., Rabouin, D., ... Goering, P. (2015). Effect of scattered-site housing using rent supplements and intensive case management on housing stability among homeless adults with mental illness: A randomized trial. JAMA, 313, 905-915.

Stergiopoulos, V., Schuler, A., Nisenbaum, R., deRuiter, W., Guimond, T., Wasylenki, D., ... Dewa, C. (2015). The effectiveness of an integrated collaborative care model vs. A shifted outpatient collaborative care model on community functioning, residential stability, and health service use among homeless adults with mental illness: A quasiexperimental study. BMC Health Services Research, 15, 348.

Tomita, A., \& Herman, D. B. (2012). The impact of Critical Time Intervention in reducing psychiatric rehospitalization after hospital discharge. Psychiatric Services, 63, 935-937.

Tomita, A., \& Herman, D. B. (2015). The role of a Critical Time Intervention on the experience of continuity of care among persons with severe mental illness after hospital discharge. The Journal of Nervous and Mental Disease, 203, 65-70.

Vandyk, A. D., Graham, I. D., Vandenkerkhof, E. G., Ross-White, A., \& Harrison, M. B. (2013). Towards a conceptual consensus of continuity in mental healthcare: Focused literature search and theory analysis. International Journal of Evidence-Based Healthcare, 11, 94109.

Wagner, J., Diehl, K., Mutsch, L., Löffler, W., Burkert, N., \& Freidl, W. (2014). Health status and utilisation of the healthcare system by homeless and non-homeless people in Vienna. Health and Social Care in the Community, 22, 300-307.

Weiser, S. D., Riley, E. D., Ragland, K., Hammer, G., Clark, R., \& Bangsberg, D. R. (2006). Brief report: Factors associated with depression among homeless and marginally housed HIV-infected men in San Francisco. Journal of General Internal Medicine, 21, 61-64. Woltmann, E., Grogan-Kaylor, A., Perron, B., Georges, H., Kilbourne, A. M., \& Bauer, M. S. (2012). Comparative effectiveness of collaborative chronic care models for mental health conditions across primary, specialty, and behavioral health care settings: Systematic review and meta-analysis. American Journal of Psychiatry, 169, 790-804. 
Table 1

Demographic and clinical characteristics of qualitative service user sample

\begin{tabular}{ll}
\hline Characteristic & $\begin{array}{l}\text { Mean } \pm \text { SD } \\
\text { Or n }(\%)\end{array}$ \\
\hline Age & $42.0 \pm 9.8$ \\
Gender & $6(27.3)$ \\
$\quad$ Woman & $16(72.7)$ \\
$\quad$ Man & \\
Education & $11(50.0)$ \\
$\quad$ Less than high school & $11(50.0)$ \\
Completed high school or attended post- & \\
$\quad$ secondary school & \\
Single longest period of homelessness & $12(54.6)$ \\
$\quad<1$ year & $5(22.7)$ \\
$\geq 1$ year to $<3$ years & $5(22.7)$ \\
$\geq 3$ years & \\
Mental health diagnoses ${ }^{1}$ & $17(77.3)$ \\
$\quad$ Mood disorder & $6(27.3)$ \\
Psychotic disorder & $9(40.9)$ \\
Anxiety disorder & $12(54.6)$ \\
Alcohol or substance misuse & \\
Comorbid medical conditions ${ }^{1}$ & $21(95.5)$ \\
$\geq 1$ conditions & $13(59.1)$ \\
$\geq 3$ conditions &
\end{tabular}

${ }^{1}$ Participants selected all that applied. 